\section{Ranolazine treatment for refractory angina in a patient with Hutchinson-Gilford progeria syndrome and end stage aortic stenosis}

\author{
Giuseppe Limongelli, ${ }^{1,2}$ \\ Emanuele Monda, ${ }^{1}$ \\ Giovanbattista Capozzi, ${ }^{1}$ \\ Martina Caiazza, ${ }^{1}$ \\ Maria Giovanna Russo ${ }^{1}$ \\ ${ }^{1}$ Unit of Pediatric Cardiology, \\ Department of Translational Sciences, \\ Luigi Vanvitelli University, AORN Colli, \\ Monaldi Hospital, Naples, Italy; \\ ${ }^{2}$ Institute of Cardiovascular Sciences, \\ University College of London, UK
}

\begin{abstract}
Management of symptoms in patients with inoperable aortic stenosis is often hard in clinical practice. We report a case of a patient with Hutchinson-Gilford progeria syndrome and end-stage aortic stenosis, considered not suitable for surgical or percutaneous approach, to whom the administration of ranolazine resulted in a considerable improvement of angina, refractory to other treatments.
\end{abstract}

\section{Introduction}

Hutchinson-Gilford progeria syndrome (HGPS) is a rare, fatal, premature aging syndrome that results from mutation in Lamin A gene. Clinical features of this disorder include short stature, craniofacial dimorphism, sclerotic skin, join contractures, bone abnormalities, alopecia, and cardiovascular disorders. ${ }^{1}$ Cardiac involvement is the commonest cause of death ${ }^{2}$ and manifests principally as coronary artery disease, valvular stenosis and hypertension. ${ }^{3,4}$

We report a case of a patient with HGPS (heterozygous p.G608G mutation in LMNA gene) and end-stage aortic stenosis to whom the administration of ranolazine resulted in a considerable improvement of angina, refractory to other treatments.

\section{Case Report}

A 15-years-old girl has been followed regularly in our division for three years, fol- lowing the echocardiographic finding of severe left ventricular hypertrophy and moderate aortic stenosis (average gradient $35 \mathrm{mmHg}$ ). Regular echocardiographic inspections showed a progressive increase in transvalvular gradient and worsening of left ventricular hypertrophy. During this period the patient reported multiple episodes of angina at rest and during exertion. For this reason, she was frequently hospitalized.

At the age of 18 , she was admitted to our division complaining cough and dyspnoea at rest. Her physical examination evidenced sinus tachycardia, peripheral swelling, jugular veins distension, liver enlargement until $3 \mathrm{~cm}$ from ribs, blood pressure 95/65 $\mathrm{mmHg}$. Echocardiography showed severe aortic stenosis (average gradient $50 \mathrm{mmHg}$ ) and left ventricular severe hypokinesia (Figures 1 and 2). At the time, she was on daily therapy with carvedilol $6.25 \mathrm{mg}$, furosemide $37.5 \mathrm{mg}$, metolazone $0.25 \mathrm{mg}$, spironolactone $10 \mathrm{mg}$, aspirin 50 $\mathrm{mg}$. She experienced a progressive worsening of angina, refractory to medical therapy. The patient underwent coronary angiography that showed no obstructive coronary artery disease to account for the angina.

Her treatment options were discussed with the heart team and, considering the fragile general condition of the patient due to the severity of the systemic phenotype and that the outcome of aortic valve dilation or replacement is not well documented, the valve disease was considered not suitable for surgical or percutaneous approach. Thus, for the purpose of improving symptoms, we introduced ranolazine $100 \mathrm{mg}$ twice a day. She tolerated it very well and within few days reported substantial improvement of angina. Angina was under control for 3 months, and no symptoms of heart failure were referred. After 3 months, she was admitted in the emergency unit and she died for a severe, untreatable heart failure.

\section{Discussion and Conclusions}

HGPS is an extremely rare condition. It is caused by a mutation in the LMNA gene which results in the production of a mutant Lamin A protein product, progerin. Death results principally due to cardiac involvement and it usually occurs between the ages of 10 and 15 years. In this syndrome, an accelerated calcific deposition was seen to occur in the aortic, coronary, cerebral, subclavian and axillary arteries, mitral annulus and aortic valve cusps. ${ }^{1}$ The onset of aortic
Correspondence: Emanuele Monda, Unit of Pediatric Cardiology, Department of Translational Sci-ences, Luigi Vanvitelli University, AORN Colli, Monaldi Hospital, Naples, Italy.

Tel.: +39.081.7062211

E-mail: emanuelemonda@me.com

Key words: Hutchinson-Gilford progeria syndrome; aortic stenosis; ranolazine.

Conflicts of interest: there are no conflicts of interest.

Received for publication: 7 October 2019

Revision received: 16 December 2019

Accepted for publication: 14 January 2020.

This work is licensed under a Creative Commons Attribution NonCommercial 4.0 License (CC BY-NC 4.0).

${ }^{\circ}$ Copyright: the Author(s), 2019

Licensee PAGEPress, Italy

Cardiogenetics 2019; 9:8609

doi:10.4081/cardiogenetics.2019.8609

stenosis is frequent in HGPS, in particular the calcific aortic valve sclerosis represents a degenerative change that is an exaggeration of normal ageing. ${ }^{3,5}$ Some of these patients develop severe aortic stenosis, often inoperable due to comorbidity, and the appearance of symptoms leads to a deterioration in the quality of life and a worse prognosis.

Ranolazine is an anti-ischemic agent used in second-line therapy for the symptomatic treatment of patient with stable angina pectoris who are inadequately controlled or intolerant to first-line antianginal therapies such as b-blockers and/or calcium antagonists.

The exact mechanism of action of ranolazine is not totally known; we know that ranolazine causes a concentration, voltage, and frequency-dependent inhibition of the late sodium current, thus preventing intracellular calcium overload during ischemia, resulting in the distribution of myocardial blood flow toward the ischemic areas with a reduction of ischemia. ${ }^{6}$

The use of ranolazine in aortic stenosis as an antianginal drug is reported in a single case report, ${ }^{7}$ which describes the efficacy of this drug on an 88-year-old lady with isolated severe aortic stenosis who was not suitable for surgical or percutaneous valve replacement due to medical comorbidity. Further studies will be needed to evaluate the possible therapeutic role of this drug in symptomatic treatment of patients with inoperable aortic stenosis. 


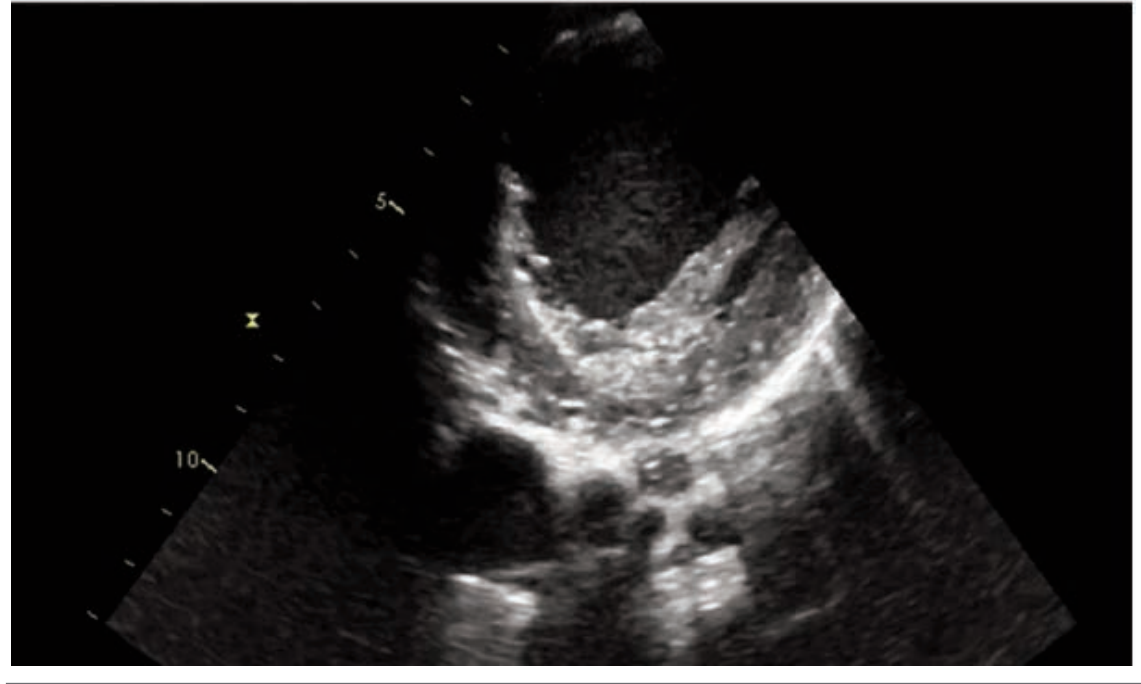

Figure 1. Severe aortic stenosis showed in parasternal short axis view.

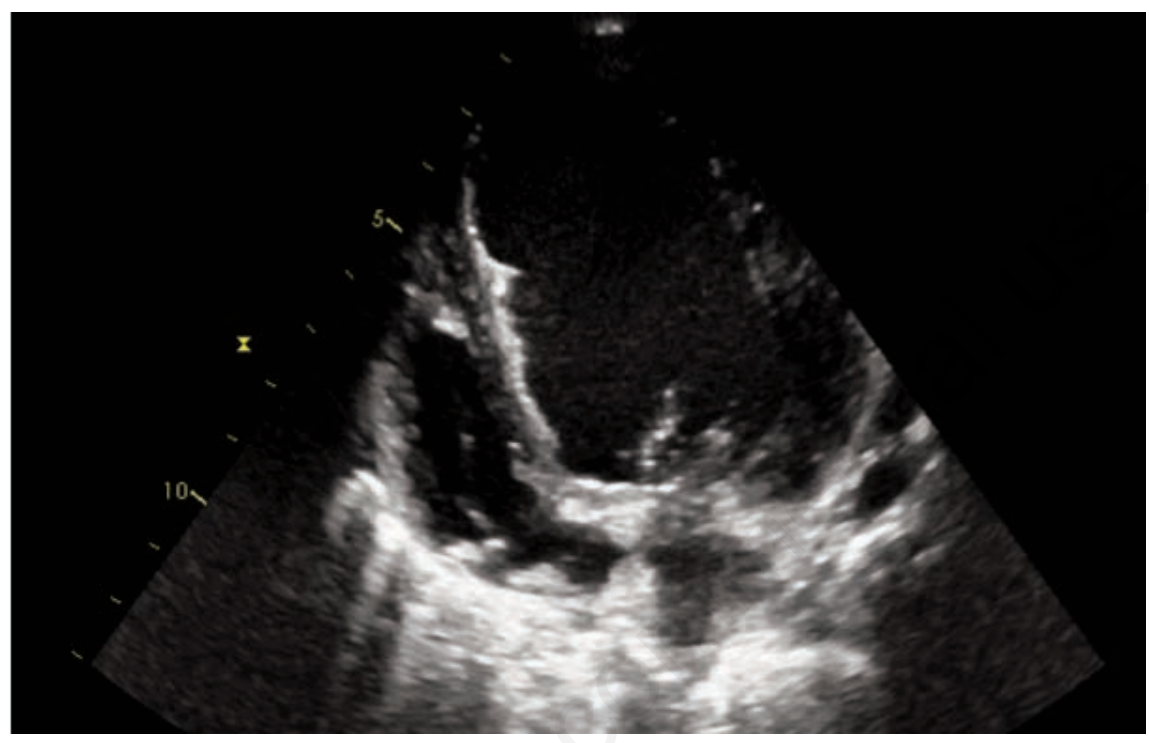

Figure 2. Severe aortic stenosis showed in apical 4-chamber view.

\section{References}

1. Badame AJ. Progeria. Arch Dermatol 1989;125:540-4.

2. DeBusk FL. The Hutchinson-Gilford progeria syndrome: Report of 4 cases and review of the literature. J Pediatr 1972;80:697-724.

3. Nair K, Ramachandran P, Krishnamoorthy KM, et al. HutchinsonGilford progeria syndrome with severe calcific aortic valve stenosis and calcific mitral valve. J Heart Valve Dis 2004; 13:866-9.

4. Makous N, Friedman S, Yakovac W, Maris EP. Cardiovascular manifestations in progeria. Report of clinical and pathologic findings in a patient with severe arteriosclerotic heart disease and aortic stenosis. Am Heart J 1962;64:334-46.

5. Merideth MA, Gordon LB, Clauss S, et al. Phenotype and course of Hutchinson-Gilford pro-geria syndrome. N Engl J Med 2008;358:592604.

6. Rayner E, Hartley, Sedlak T. Ranolazine: a contemporary review. J Am Heart Assoc 2016;5:e003196.

7. Athauda-Arachchi P, Lang C. Metabolic antianginal agent ranolazine offers good symptom re-lief in a patient with inoperable severe aortic stenosis. Cardiovasc Ther 2012;30:e210-1. 